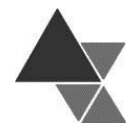

\section{Estado nutricional de crianças e adolescentes de um município do interior paulista e vínculo com programas de transferência de renda ${ }^{1}$}

\author{
Maria Julia de Miguel Amistá ${ }^{2}$ e Marina Vieira da Silva ${ }^{3}$
}

O objetivo deste trabalho foi conhecer o perfil socioeconômico e o estado nutricional de alunos beneficiários do Programa Nacional de Alimentação Escolar inseridos em famílias cadastradas em programas de transferência de renda e a situação de segurança alimentar dos domicílios. Trata-se de um estudo transversal realizado com 425 alunos matriculados em escolas públicas municipais de Guariba, São Paulo. Para a avaliação nutricional foram consideradas as variáveis antropométricas, peso e altura, e o Índice de Massa Corporal (IMC). Para a investigação da situação de Segurança Alimentar utilizou-se a Escala Brasileira de Medida da Insegurança Alimentar - EBIA. Os resultados indicaram que 37,65\% das famílias estavam inscritas em algum programa de transferência de renda. Dos alunos vinculados a estes tipos de programas, $61,25 \%$ foram classificados como eutróficos. O excesso de peso foi prevalente naqueles, cujos rendimentos eram inferiores a R \$600,00 (38,96\%). Entre as famílias classificadas em situação de Segurança Alimentar, 28,17\% estavam vinculadas a programas de transferência de renda e apenas $5 \%$ das famílias com este tipo de vínculo eram atingidas pela Insegurança Alimentar grave. Os resultados indicam que existe uma tendência de adequação tanto do estado nutricional quanto da Segurança Alimentar entre aqueles que recebem benefício monetário vinculado aos programas de transferência direta de renda.

Palavras-chave: Estado nutricional, Segurança alimentar e Nutricional, Vulnerabilidade social.

\section{Nutritional status of children and adolescents in a city in the São Paulo estate and bond with cash transfer programs}

The objective of this study was to identify the socioeconomic profile, the nutritional status of beneficiaries students of the National School Meals Program entered into families enrolled in income cash transfers of public programs and food security situation of households. It is a cross-sectional study of 425 students enrolled in public schools in Guariba, São Paulo. For nutritional assessment were considered the anthropometric variables, weight and height, and Body Mass Index (BMI). To research the food security situation was used the Brazilian Scale of Food Insecurity and Hunger - EBIA. The results indicated that $37.65 \%$ of families were enrolled in a cash transfer program. Students linked to these types of programs, $61.25 \%$ were classified as eutrophic. Being overweight was prevalent in those whose incomes were less than R $\$ 600.00$ (38.96\%). Among households classified in food security situation, $28.17 \%$ were linked to income transfer programs and only $5 \%$ of the families with this type of bond were affected by severe food insecurity. The results indicate that there is a tendency to adapt both the nutritional status as the Food Security among those receiving monetary benefit related to direct cash transfers of public programs.

Key-words: Nutritional status, Food security, Social vulnerability.

\footnotetext{
${ }^{1} \mathrm{O}$ artigo é baseado na dissertação da autora intitulada "Programa de alimentação escolar: perfil dos beneficiários, qualidade e atuação de gestores e da comunidade de Guariba", Escola Superior de Agricultura Luiz de Queiroz, 2013.

${ }^{2}$ Doutoranda. Programa de Pós-graduação em Ciências e Tecnologia de Alimentos - Escola Superior de Agricultura "Luiz de Queiroz" - ESALQ/USP. Av. Pádua Dias, no 11 Cx. Postal 9 - Piracicaba - SP - CEP 13418-900. E-mail: amistamjm@usp.br

${ }^{3}$ Professora Doutora. Departamento de Agroindústria, Alimentos e Nutrição. Escola Superior de Agricultura "Luiz de Queiroz" - ESALQ/USP.
} 


\section{INTRODUÇÃO}

O direito humano à alimentação e ao acesso aos alimentos constitui-se como um direito social garantido pela Constituição Brasileira e são requisitos básicos para a promoção e proteção da saúde da população. Cabe ao Estado proteger o direito dos indivíduos no que tange uma alimentação saudável, nutricionalmente adequada e associar medidas e ações que visem prover condições quando esta situação esteja inviabilizada, seja por desastres naturais ou limitações monetárias, de modo a garantir que a comunidade tenha capacidade de produzir ou adquirir alimentação segura e de qualidade $e^{[1]}$.

De acordo o artigo 3 da Lei Orgânica de Segurança Alimentar e Nutricional (LOSAN), Lei no 1.346 de 15 de setembro de 2006, a Segurança Alimentar e Nutricional fundamenta-se no direito de todos ao acesso permanente e regular de alimentos em quantidade suficiente e de qualidade que respeitem a diversidade cultural e econômica, até no que se refere o comprometimento ao acesso a outras necessidades essenciais ao indivíduo e que sejam ambientalmente e socialmente sustentáveis[ ${ }^{[2]}$. Diante desta concepção o conceito de Segurança Alimentar e Nutricional foi reafirmado, fortalecido e ampliado com o propósito de melhorar as condições de alimentação, nutrição e saúde da população brasileira tomando forma nas políticas públicas na área em todo o país.

Dentre os programas de políticas de alimentação no Brasil, destaca-se o Programa Nacional de Alimentação Escolar (PNAE). Criado na década de 1.950 é considerado um dos maiores e mais duradouro programa na área e constitui-se em uma política pública de abrangência nacional pautada no oferecimento de refeições a estudantes do ensino básico da rede pública de educação, cujo objetivo é fornecer, no mínimo, $30 \%$ das necessidades nutricionais diárias de modo a contribuir para o crescimento e desenvolvimento biopsicossocial dos alunos bem como para a melhoria do rendimento escolar ${ }^{[3]}$. Em sua gênese, sua essência era de um programa de caráter suplementar e assistencial com o propósito de combater os problemas endêmicos de fome e subnutrição muito associados ao contexto social e econômico a qual a população brasileira estava inserida à época. Atualmente, porém, devido às mudanças ocorridas nos padrões alimentares de crianças e adolescentes, deve-se reconhecer a importância do programa de alimentação escolar quanto ao seu papel social e de Educação Alimentar e Nutricional no que tange a Segurança Alimentar e Nutricional.

Outro programa social de abrangência nacional que possui caráter de seguridade alimentar à população brasileira é o Programa Bolsa Família (PBF). Instituído pelo governo federal por meio da unificação dos programas Bolsa Escola, Bolsa Alimentação, Auxílio Gás e Cartão Alimentação, trata-se de um programa de transferência direta de renda com recursos recursos governamentais que atendem famílias que se encontram em situação de vulnerabilidade e risco social ${ }^{[4]}$. Dentre as condicionalidades exigidas pelo programa o acompanhamento nutricional e o acompanhamento da saúde ganham destaque e abrem lacunas no sentido de responder aos questionamentos relativos ao alcance da influência que este tipo de programa é capaz de exercer sobre a alimentação e nutrição das famílias por ele beneficiadas.

Diante da perspectiva do impacto positivo dos programas de transferência de renda no estado nutricional e levando-se em conta a hipótese de que o recurso monetário oferecido às famílias inscritas nestes tipos de programas poderia atuar sobre a segurança alimentar e nutricional de seus beneficiários, os objetivos deste trabalho foram: conhecer o perfil socioeconômico, o estado nutricional de alunos beneficiários do PNAE inseridos em famílias cadastradas em programas de transferência de renda e a situação de segurança alimentar e nutricional das famílias beneficiárias.

\section{MATERIAL E MÉTODOS}

Trata-se de um estudo do tipo transversal, realizado entre fevereiro e junho de 2.013, tendo uma amostra inicial composta $(n=425)$ por alunos matriculados no ensino fundamental (do $2^{\underline{0}}$ ao $9^{\underline{0}}$ ano) da rede pública de ensino municipal de Guariba, estado de São Paulo, usuários do Programa Nacional de Alimentação Escolar. O município de Guariba está localizado no interior paulista na região administrativa de Ribeirão Preto e possui suas bases econômicas apoiadas nas atividades agropecuárias, pautada principalmente na cultura da cana de açúcar e no setor sucroalcooleiro. Apresenta uma alta taxa de urbanização $(97,92 \%)$, um índice de desenvolvimento humano municipal (IDH-M) de 0,719 e renda média per capita de $\mathbf{R} \$ 671,34{ }^{[5]}$. 
Para a avaliação socioeconômica e da situação de Segurança Alimentar e Nutricional os pais ou responsáveis preencheram um questionário a eles destinados cujas questões buscavam angariar informações sobre o contexto social e econômico em que o aluno está inserido. No tocante à situação de Segurança Alimentar no domićlilio optou-se pelo uso de um instrumento que mensura diretamente a situação de Segurança e Insegurança Alimentar no domicílio, a Escala Brasileira de Medida da Insegurança Alimentar (EBIA) ${ }^{[6]}$.

\section{Avaliação do Estado Nutricional}

Para a avaliação nutricional dos alunos foram consideradas as variáveis antropométricas, peso e altura, e o Índice de Massa Corporal (IMC) o qual é definido pela a relação entre o peso $(\mathrm{kg})$ e a altura (m) elevada ao quadrado $\left(\mathrm{kg} / \mathrm{m}^{2}\right)$. O peso foi aferido uma única vez, utilizando balança da marca Filizola ${ }^{\circledR}$ devidamente calibrada, com capacidade de até 150 quilogramas e sensibilidade de 0,5 quilogramas, sendo que os alunos no momento da pesagem trajavam vestimentas leves e estavam descalços. A altura foi aferida também uma única vez, com auxílio de estadiômetro presente na balança, com escala em milímetros, estando descalços, sem meias, com pés e tornozelos unidos e postura ereta, tendo a barra horizontal do equipamento posicionada firmemente sobre a cabeça. Alunos que apresentavam impossibilidade ter sua altura ou peso aferidos, por quaisquer motivos de limitações, não entraram na amostra. Para a interpretação do IMC foi utilizado o escore $Z$ do IMC, calculados pelo programa para computador em World Health Organization (2007) ${ }^{[7]}$. Os alunos que apresentaram escore $Z \leq-2$ foram classificados como magros (IMC abaixo do esperado para a idade), aqueles identificados no intervalo $-2<Z<1$ foram classificados como eutróficos (IMC compatível para idade) e aqueles classificados com escore $Z$ de IMC entre os intervalos $1 \leq Z<2$ e $Z \geq 2$ foram diagnosticados com sobrepeso e obesidade, respectivamente ${ }^{[8]}$.

As variáveis para este estudo foram idade, sexo, peso, altura e vínculo a programas de transferência de renda. Os instrumentos metodológicos adotados foram: questionário de avaliação antropométrica (peso e altura) e questionário socioeconômico auto-administrado, previamente validado, para os pais dos alunos amostrados.
Para a análise dos dados foram elaboradas tabelas para a viabilização dos testes de associações de variáveis selecionadas envolvendo o qui-quadrado comum. As análises foram elaboradas com a adoção dos recursos do software Statistical Analysis System$S A S^{[9]}$.

\section{Aspectos Éticos}

A fim de atender o que estabelece a Resolução no 196, de 10 de outubro de 1.996, do Conselho Nacional de Saúde, este estudo foi submetido ao Comitê de Ética da Escola Superior de Agricultura "Luiz de Queiroz" (ESALQ Piracicaba, SP), Universidade de São Paulo, com o Protocolo no 99 e aprovado em 14 de junho de 2.012. Os alunos participantes só foram avaliados após esclarecimento aos pais ou responsáveis sobre o estudo e assinatura do Termo de Consentimento Livre e Esclarecido.

Foram obtidas autorizações do representante do Poder Executivo bem como da secretária da Educação do município no ano de 2.012 e de todos os diretores das escolas que integraram a amostra.

\section{RESULTADOS}

Participaram do estudo 425 alunos de ambos os sexos, sendo 215 meninas $(50,59 \%)$ e 210 meninos (49,41\%), com idades entre sete e 16 anos, matriculados em seis escolas da rede municipal de ensino, as quais foram sorteadas para compor a amostra, de maneira que a probabilidade de uma escola ser incluída na amostra fosse proporcional ao número de alunos.

Dentre os alunos avaliados, 308 (72,47\%) tiveram a renda familiar informada pelo pai ou responsável. A renda per capita de 90,91\% das famílias não alcançava $\mathrm{R} \$ 600,00$.

$\mathrm{Na}$ Tabela 1 encontra-se a distribuição dos alunos de acordo com o estado nutricional e os rendimentos familiares per capita. 
Tabela 1: Distribuição dos alunos da rede pública municipal de ensino de acordo com o estado nutricional (com base nos escores $Z$ de IMC) e os estratos de rendimentos familiares. Guariba - 2013.

\begin{tabular}{|c|c|c|c|c|c|c|c|c|c|c|}
\hline \multirow{3}{*}{$\begin{array}{c}\text { Estratos de } \\
\text { rendimentos familiares } \\
\text { per capita (em Reais) }\end{array}$} & \multirow{2}{*}{\multicolumn{2}{|c|}{ Observações }} & \multicolumn{8}{|c|}{ Estado Nutricional } \\
\hline & & & \multicolumn{2}{|c|}{$Z \leq-2$} & \multicolumn{2}{|c|}{$-2<Z<1$} & \multicolumn{2}{|c|}{$1 \leq Z<2$} & \multicolumn{2}{|c|}{$Z \geq 2$} \\
\hline & $n$ & $\%$ & $n$ & $\%$ & $n$ & $\%$ & $n$ & $\%$ & $n$ & $\%$ \\
\hline \multirow{3}{*}{$\leq 120,00$} & 36 & 11,69 & 0 & 0,00 & 19 & 6,17 & 8 & 2,60 & 9 & 2,92 \\
\hline & & & & & & $(11,38)$ & & $(11,94)$ & & $(12,68)$ \\
\hline & & & & & & {$[52,78]$} & & {$[22,22]$} & & {$[25,00]$} \\
\hline \multirow{3}{*}{$120,00--\mid 240,00$} & 109 & 35,39 & 2 & 0,65 & 72 & 23,38 & 17 & 5,52 & 18 & 5,84 \\
\hline & & & & $(66,67)$ & & $(43,11)$ & & $(25,37)$ & & $(25,35)$ \\
\hline & & & & {$[1,83]$} & & {$[66,06]$} & & {$[15,60]$} & & {$[16,51]$} \\
\hline \multirow{3}{*}{$240--\mid 360$} & 64 & 20,78 & 1 & 0,32 & 32 & 10,39 & 17 & 5,52 & 14 & 4,55 \\
\hline & & & & $(33,33)$ & & $(19,16)$ & & $(25,37)$ & & $(19,72)$ \\
\hline & & & & {$[1,56]$} & & {$[50,00]$} & & {$[26,56]$} & & {$[21,88]$} \\
\hline \multirow{3}{*}{$360--\mid 600$} & 71 & 23,05 & 0 & 0,00 & 34 & 11,04 & 18 & 5,84 & 19 & 6,17 \\
\hline & & & & & & $(20,36)$ & & $(26,87)$ & & $(26,76)$ \\
\hline & & & & & & {$[47,88]$} & & {$[25,35]$} & & {$[26,77]$} \\
\hline \multirow{3}{*}{$>600$} & 28 & 9,09 & 0 & 0,00 & 10 & 3,25 & 7 & 2,27 & 11 & 3,57 \\
\hline & & & & & & $(5,99)$ & & $(10,45)$ & & $(15,49)$ \\
\hline & & & & & & {$[35,71]$} & & {$[25,00]$} & & {$[39,29]$} \\
\hline Total & 308 & 100,00 & 3 & 0,97 & 167 & 54,23 & 67 & 21,75 & 71 & 23,05 \\
\hline
\end{tabular}

$\chi^{2}=16,59$, com 12 graus de liberdade, não-significativo.

Nota: os números entre parênteses são os percentuais em relação aos valores totais observados nas colunas.

Os números entre colchetes são os percentuais em relação aos valores totais observados nas linhas. Análises elaboradas tendo por base os dados fornecidos por 308 pais/responsáveis, para os quais foram obtidas informações válidas.

Fonte: Autoria própria.

Em 55,53\% das famílias a mãe era a responsável pelo aluno. Quanto à ocupação dos pais ou responsáveis, $65,69 \%$ realizavam algum tipo de atividade remunerada e $15,76 \%$ estavam desempregados. Entre aqueles que exerciam atividade remunerada, apenas $50,12 \%$ trabalhavam com registro em carteira profissional. Quanto à escolaridade dos pais ou responsáveis 1,41\% nunca frequentaram a escola, $3,76 \%$ nunca frequentaram a escola, porém sabem ler e escrever e $82,35 \%$ possuem até oito anos de estudo. Apenas 2,12\% dos pais ou responsáveis cursaram o ensino superior.

Com relação às características relativas ao saneamento básico, $98,82 \%$ das famílias residem em áreas que possuem coleta pública de lixo regular, 93,88\% tem acesso à rede pública de esgoto, 92,72\% possuem as instalações sanitárias dentro da própria residência, $88 \%$ tem a rua de casa asfaltada e/ou com paralelepípedo e $88,94 \%$ tem acesso ao abastecimento de água da rede pública.

No que se refere o vínculo a programas assistenciais, $37,65 \%$ estavam inscritas em algum programa de transferência condicionada de renda.
Das famílias que informaram possuir este tipo de vínculo, 80,62\%, eram beneficiárias do Programa Bolsa Família. Outros programas citados foram Renda Cidadã $(n=10)$, Viva Leite $(n=2)$, Ação Jovem $(n=1)$, Auxílio Reclusão $(n=1)$, ProJovem $(n=1)$, Bolsa Escola $(n=1)$, Auxílio Desemprego $(n$ $=1)$ e Amparo Social $(n=1)$, todos eles vinculados à Secretaria de Ação Social do município. Mais da metade das famílias $(51,25 \%)$ informaram receber benefícios entre $\mathrm{R} \$ 100$ e $\mathrm{R} \$ 200 ; 16,25 \%$ recebiam valores inferiores a $\mathrm{R} \$ 100,17,5 \%$ valores acima de $\mathrm{R} \$ 200$ e $15 \%$ omitiram os valores recebidos.

Os 425 alunos tiveram seu estado nutricional avaliado. Da totalidade, a proporção de alunos diagnosticados com escore $Z \leq-2$ foi de $0,94 \%$, distribuídos de forma similar entre o sexo feminino e masculino $(0,47 \%)$. Mais da metade dos alunos $(52,71 \%)$ foram considerados eutróficos (integram o intervalo indicativo de IMC adequado para a idade: $-2<Z<1)$ e $46,35 \%$ dos alunos foram classificados com excesso de peso, sendo $22,35 \%$ identificados com sobrepeso $(1 \leq Z<2)$ e $24 \% \mathrm{com}$ obesidade $(Z \geq 2)$. O sobrepeso apresentou-se maior para o sexo masculino $(12,71 \%)$ enquanto que 
a obesidade foi maior para o grupo do sexo feminino (12,47\%). Não foram observadas diferenças significativas entre meninos e meninas quanto ao diagnóstico do estado nutricional.
A Tabela 2 mostra a distribuição dos alunos de acordo com o vínculo a programas de transferência de renda e estado nutricional.

Tabela 2: Distribuição dos alunos da rede pública municipal de ensino de acordo com o vínculo a programas de transferência de renda e estado nutricional (com base nos escores $Z$ de IMC). Guariba - 2013.

\begin{tabular}{|c|c|c|c|c|c|c|c|c|c|c|}
\hline \multirow{3}{*}{$\begin{array}{c}\text { Vínculo a programas } \\
\text { de transferência de } \\
\text { renda }\end{array}$} & \multirow{2}{*}{\multicolumn{2}{|c|}{ Observações }} & \multicolumn{8}{|c|}{ Estado Nutricional } \\
\hline & & & \multicolumn{2}{|c|}{$Z \leq-2$} & \multicolumn{2}{|c|}{$-2<Z<1$} & \multicolumn{2}{|c|}{$1 \leq Z<2$} & \multicolumn{2}{|c|}{$Z \geq 2$} \\
\hline & $n$ & $\%$ & $n$ & $\%$ & $n$ & $\%$ & $n$ & $\%$ & $n$ & $\%$ \\
\hline $\operatorname{Sim}$ & 160 & 37,65 & 0 & 0,00 & 98 & $\begin{array}{l}23,06 \\
(43,75) \\
{[61,25]}\end{array}$ & 35 & $\begin{array}{c}8,23 \\
(36,84) \\
{[21,87]}\end{array}$ & 27 & $\begin{array}{c}6,35 \\
(26,47) \\
{[16,88]}\end{array}$ \\
\hline Não & 249 & 58,59 & 4 & $\begin{array}{r}100,00 \\
(0,94) \\
{[1,61]}\end{array}$ & 115 & $\begin{array}{l}27,06 \\
(51,34) \\
{[46,18]}\end{array}$ & 58 & $\begin{array}{l}13,65 \\
(61,05) \\
{[23,29]}\end{array}$ & 72 & $\begin{array}{l}16,94 \\
(70,59) \\
{[28,92]}\end{array}$ \\
\hline $\begin{array}{l}\text { Não sabe/ } \\
\text { informado }\end{array}$ & 16 & 3,76 & 0 & 0,00 & 11 & $\begin{array}{r}2,59 \\
(4,91) \\
{[68,75]}\end{array}$ & 2 & $\begin{array}{r}0,47 \\
(2,11) \\
{[12,50]}\end{array}$ & 3 & $\begin{array}{r}0,71 \\
(2,94) \\
{[18,75]}\end{array}$ \\
\hline Total & 425 & 100,00 & 4 & 0,94 & 224 & 52,71 & 95 & 22,35 & 102 & 24,00 \\
\hline
\end{tabular}

Nota: os números entre parênteses são os percentuais em relação aos valores totais observados nas colunas.

Os números entre colchetes são os percentuais em relação aos valores totais observados nas linhas.

Fonte: Autoria própria.

Pouco mais da metade das famílias inserem-se em um contexto de Segurança Alimentar (50,12\%). Quanto à situação de Insegurança Alimentar, $37,17 \%$ caracteriza-se como leve, 9,18\% moderada e $3,53 \%$ como grave. Das famílias que forneceram as informações válidas relativas à renda familiar, $47,09 \%$ estão em situação de Segurança Alimentar, $38,96 \%$ em Insegurança leve, 10,38\% moderada e $3,57 \%$ grave. Das famílias que se encontram em situação de Insegurança Grave, 90,90\% possuem renda per capita menor que $\mathrm{R} \$ 600,00$. Entre àquelas classificadas em situação de Segurança Alimentar, $28,17 \%$ estão vinculadas a programas de transferência de renda. Das famílias identificadas como expostas à Insegurança Alimentar leve, $44,30 \%$ estão vinculadas a programas de transferência de renda, enquanto que para a Insegurança moderada este número é proporcionalmente maior, atingindo $56,42 \%$ das famílias beneficiárias. A Insegurança Alimentar grave atinge apenas $5 \%$ das famílias com vínculo a programas de transferência de renda, porém, da totalidade das famílias atingidas pela Insegurança grave 53,33\% são beneficiadas por estes tipos de programas. Foi captada associação significativa entre as variáveis.

\section{DISCUSSÃO}

Diante da situação socioeconômica apresentada pelas famílias, devem ser destacados os rendimentos per capita, inferiores a um salário mínimo vigente à época $(\mathrm{R} \$ 678,00)$. Segundo dados da Fundação SEADE ${ }^{[10]}$, a renda domiciliar média no ano de 2.010, para a população de Guariba era $\mathrm{R} \$ 1.927,00$ e em $18,7 \%$ dos domićlios do município a renda per capita não ultrapassava meio salário mínimo. Ao distribuir a população do município de acordo com o Índice Paulista de Vulnerabilidade Social (IPVS), a maior proporção $(37,3 \%)$ se enquadra no Grupo 4, vulnerabilidade média, com rendimento nominal médio dos domicílios de $\mathrm{R} \$ 1.836,00$ e em 18,9\% não ultrapassa meio salário mínimo per capita. A desigualdade de renda diminuiu no município entre os anos de 2.000 e 2.010. O Índice de Gini, o qual é usado para medir o grau de concentração de renda por apontar a diferença entre os rendimentos dos mais pobres e dos mais ricos, passou de 0,50 para 0,43. Essa diminuição na desigualdade social do município deve-se em grande parte ao aumento gradativo do número de famílias beneficiadas no município por programas de transferência condicionada de renda no período. $\mathrm{O}$ Programa Bolsa Família, por exemplo, atendia no ano de 2.013, 1.613 famílias correspondendo a $15,78 \%$ da população do município. No período de 2.004 a 2.012, a soma dos recursos transferidos pelo PBF teve um aumento de $2.061,72 \%{ }^{[11]}$. 
Os resultados indicam que possivelmente o excesso de consumo tem causado mais repercussões (negativas) no estado nutricional do que a insuficiência alimentar nesta população amostral, uma vez que $0,94 \%$ dos alunos apresentaram-se magros e 46,35\% com excesso de peso. Ao analisar os resultados quanto à distribuição dos alunos de acordo com o estado nutricional e os rendimentos familiares per capita, nota-se que o excesso de peso concentra-se nos estratos com rendimentos inferiores a $\mathrm{R} \$ 600,00$ (38,96\%), distribuídos de forma similar para sobrepeso e obesidade $(19,48 \%)$. Proporção de $89,55 \%$ e $84,51 \%$ dos alunos classificados com sobrepeso e obesidade, respectivamente, estão inseridos em famílias que recebem até $\mathrm{R} \$ 600,00$ per capita. Dos alunos inseridos em famílias com rendimentos per capita acima de $\mathrm{R} \$ 600,00,35,71 \%$ foram diagnosticados como eutróficos, $25 \%$ com sobrepeso e $39,29 \%$ com obesidade. Estes resultados merecem destaque, uma vez que praticamente nove em cada dez alunos com sobrepeso integram famílias socialmente vulneráveis. Estudos com as mesmas variáveis indicam o aumento da prevalência do sobrepeso e da obesidade infantil em famílias de baixa renda, atribuídos possivelmente a ampliação do consumo de alimentos ricos em gorduras e carboidratos simples, seja pela falta de conhecimento sobre alimentação saudável ou pelo fator monetário, que muitas vezes impossibilita a aquisição de alimentos de alto valor nutricional ou que define a compra de alimentos mais acessíveis a este estrato populacional, porém de baixo valor nutricional ${ }^{[12-14]}$.

Ao analisar as variáveis, estado nutricional e vínculo a programas de distribuição de renda, foi possível captar relação estatisticamente significativa. Entre os alunos classificados com $Z \leq-2(n=4)$, nenhum mantinha vínculo com programas de transferência de renda. Mais da metade $(61,25 \%)$ dos alunos beneficiados pelos programas foram classificados no intervalo $-2<Z<1$, indicativo de eutrofia. Dos diagnosticados com excesso de peso, $14,58 \%$ recebiam algum tipo de benefício $(8,23 \%$ classificados com sobrepeso e 6,35\% com obesidade). Estar vinculado a um programa de distribuição de renda mostrou-se um fator positivo para o adequado estado nutricional do aluno. Este fato pode estar relacionado com as exigências que os programas trazem em suas condicionalidades no quesito saúde, como é o caso do PBF, em que todas as crianças uma vez ao mês devem passar pelo posto de saúde para ter o peso e altura aferidos. Esta condicionalidade acontece no município de Guariba para crianças até os sete anos de idade e pode caracterizar-se como um fator inibidor do desenvolvimento do excesso de peso nesta população.

Em estudo com 22.927 crianças de 419 municípios de 23 estados brasileiros menores de cinco anos de idade inseridas em famílias que recebiam benefício de renda por meio do PBF, os autores concluíram que as exigências que os beneficiários de programas de transferência de renda, especificamente do Programa Bolsa Família, precisam cumprir os induzem a procurar mais o serviço de saúde e a priorizar a compra de alimentos para as crianças, fato que pode explicar, em parte, a adequação nutricional neste grupo em questão. Além disso, os resultados obtidos pelos autores apontam que o PBF garante às famílias maior acesso a bens e serviços que os conduzam à melhoria da nutrição das crianças ${ }^{[15]}$.

Quanto à Segurança Alimentar e Nutricional (SAN), os resultados apresentados para a situação de Segurança Alimentar para as famílias pesquisadas são abaixo da média nacional $(69,8 \%)$ e a situação de Insegurança Alimentar são superiores aos encontrados para a macrorregião na qual o município de Guariba se situa (23,3\%, sendo a Insegurança grave menor que 3\%). Estudo realizado no município de Duque de Caxias, no Rio de Janeiro, utilizando-se a EBIA como instrumento para a avaliação da Segurança Alimentar, mostrou que em famílias com renda per capita mensal inferior a um quarto de salário mínimo (vigente à época), apenas $12 \%$ foram classificadas na categoria "Segurança Alimentar", enquanto 21,3\% integravam o estrato "Insegurança Alimentar Grave". Com o acréscimo de renda, a situação de Segurança Alimentar aumentou e de Insegurança reduziu ${ }^{[16]}$. Hoffmann $^{[17]}$ ao analisar a insegurança alimentar no Brasil utilizando os dados da Pesquisa Nacional por Amostra de Domicílios (PNAD) de 2004, 2009 e 2013, tendo como base a Escala Brasileira de Medida de Insegurança Alimentar (EBIA), verificou que a insegurança alimentar dos domićlios brasileiros diminuiu ao longo dos anos e que a renda domiciliar per capita pode ser considerada o principal determinante da segurança alimentar, uma vez que a insegurança alimentar apresenta-se fortemente associada a baixos níveis de renda domiciliar per capita. No mesmo estudo foi possível também captar uma tendência à insegurança alimentar maior quando a renda domiciliar per capita é instável.

No que se refere ao acesso à alimentação dos beneficiários do PBF, dados mostram que ocorreram 
melhorias na qualidade (em menor proporção) e na quantidade dos gêneros consumidos, uma vez que famílias que não tinham alimentação básica suprida começaram a adquirir mais alimentos considerados como básicos, como por exemplo, o arroz e o feijão, além do aumento expressivo de carnes $(61 \%)^{[4]}$. Diante do exposto, pode-se sugerir que o incremento na renda familiar vindo de programas de transferência de renda pode amenizar a situação de Insegurança Alimentar de uma dada população, uma vez que a renda per capita está significativamente associada à SAN.

\section{CONCLUSÃO}

Por meio dos resultados apresentados, podese notar que existe uma tendência de adequação tanto do estado nutricional quanto da Segurança Alimentar entre aqueles que recebem benefício monetário vinculado aos programas de transferência direta de renda. A Segurança Alimentar e Nutricional é um processo multifatorial o qual possui vários fatores inter-relacionados que contribuem para o seu alcance, devendo-se considerar muito além do não acesso à alimentação pelo déficit de renda; porém, não há como desconsiderar o impacto positivo que programas de transferência de renda possam agregar às famílias beneficiadas por eles. Face ao exposto, este estudo pode contribuir para o início de novas análises na área que contribuam para um melhor entendimento sobre a qualidade da alimentação de crianças e adolescentes beneficiários destes programas bem como subsidiar a elaboração de programas e políticas públicas no tocante à Segurança Alimentar e Nutricional dos munícipes. Estudos longitudinais que sejam destinados a avaliar o impacto de programas de transferência de renda sobre $O$ consumo alimentar deste mesmo grupo em questão também se fazem necessários.

\section{REFERÊNCIAS}

[1] Ministério da Saúde. Política Nacional de Alimentação e Nutrição [Internet]. Brasília, 2013 [Acesso em 12 nov 2013]. 83 p. Disponível em: <http://bvsms.saude.gov.br/bvs/publicacoes/politica_na cional_alimentacao_nutricao.pdf $>$

[2] BRASIL. Cria o Sistema Nacional de Segurança Alimentar e Nutricional - SISAN com vistas em assegurar o direito humano à alimentação adequada e dá outras providências. Lei no 11.346 , de 15 de setembro 2006.

[3] BRASIL. Ministério da Educação. Fundo Nacional de Desenvolvimento da Educação. Dispõe sobre o atendimento da alimentação escolar aos alunos da educação básica no âmbito do Programa Nacional de Alimentação Escolar - PNAE. Resolução/CD/FNDE no 26, de 17 de junho de 2013.

[4] Instituto Brasileiro de Análises Sociais e Econômicas. Repercussões do Programa Bolsa Família na segurança alimentar e nutricional das famílias beneficiadas: documento síntese. Rio de Janeiro, 2008. 20p.

[5] Programa das Nações Unidas Para o Desenvolvimento no Brasil. Atlas do Desenvolvimento Humano no Brasil 2013 [Internet]. Brasília: PNUD: 2013[Acesso em: 05 ago 2013]. Disponível em: <http://www.atlasbrasil.org.br/2013/>

[6] Segall-Corrêa AM, Marin-Leon L. A segurança alimentar no Brasil: proposição e usos da Escala Brasileira de Medida da Insegurança Alimentar (EBIA) de 2003 a 2009. Segur Aliment Nutr. 2009:16(2):1-19. Disponível em:

<http://periodicos.sbu.unicamp.br/ojs/index.php/san/a rticle/view/8634782>

[7] World Health Organization [Internet]. Geneva: WHO; c2013. Growth reference 5-19 years: macros; [Acesso em: 27 jul 2013]; [1 arquivo 10 documentos]. (Arquivo "Download SAS macro") Disponível em: $<$ http://www.who.int/growthref/tools/en/>

[8] World Health Organization. WHO Child Growth Standards: length/height-for-age, weight-for-age, weightfor-length, weight-for-height and body mass index-forage: methods and development. Geneva: WHO; 2006. $312 \mathrm{p}$.

[9] SAS Institute Inc. The Statistical Analisys [CD-ROM]. System.Versão 9.0. Cary: SAS; 2004. 1 CD-ROM.

[10] Assembléia Legislativa do Estado de São Paulo [nternet]. São Paulo: SEADE; c2013. Indice Paulista de Responsabilidade Social e Indice Paulista de Vulnerabilidade Social: Guariba. 2012 [Acesso em: 01 jun 2013]. Disponível em: < http://indices-ilp.al.sp.gov.br/>

[11] Controladoria-Geral da União: Portal de Transparência: em gráficos [Internet]. Brasília: Portal da Transparência; c2013. Programa Bolsa Família do Governo Federal: distribuição por UF - Recursos transferidos ano a ano - SP. out 2015 [Acesso em: 01 jul 2013]. Disponível em <http://www.portaldatransparencia.gov.br/graficos/bols afamilia/>

[12] Motta MEFA, Silva GAP. Desnutrição e obesidade em crianças: delineamento do perfil de uma comunidade de baixa renda. J Pediatr. 2001:77(4):288-93. 
[13] Costa MB, Silva JHA, Simões ACSR, et al. Obesidade infantil: características em uma população atendida pelo programa de saúde da família. Rev APS. 2011:14(3):283-8.

[14] Silva VP, Zurita RCM. Prevalência dos fatores de risco da obesidade infantil nos centros municipais de educação infantil do município de Maringá-PR 2010. Rev Saúde Pesq. 2012:5(1):9-25.

[15] Paes-Sousa R, Santos LMP, Miazaki ES. Effects of a conditional cash transfer programme on child nutrition in Brazil. Bull World Health Org [Internet]. 2011 [Acesso em: 28 set 2015]:89(7):496-503. Disponível em: <http://www.who.int/bulletin/volumes/89/7/10-

084202/en/>

[16] Salles-Costa R, Pereira RA, Vasconcellos MTL, et al. Associação entre fatores socioeconômicos e insegurança alimentar: estudo de base populacional na Região Metropolitana do Rio de Janeiro, Brasil. Rev Nutr Campinas. 2008:21(1 Supl):99-109.

[17] Hoffmann R. Brasil, 2013: mais segurança alimentar. Segur Aliment Nutr [internet]. 2014 [Acesso em: 28 set 2015]:21(2):422-36. Disponível em: <http://periodicos.sbu.unicamp.br/ojs/index.php/san/a rticle/view/8634472> 